\title{
CUIDADOS DE ENFERMAGEM A PESSOA IDOSA EM TEMPOS DE PANDEMIA COVID-19
}

Bárbara Belmonte Bedin; Universidade Regional Integrada do Alto Uruguai e das Missões- Campus de Santiago, RS; barbarabbedin@hotmail.com

Diulia Molazzane Gabert; Universidade Regional Integrada do Alto Uruguai e das Missões- Campus de Santiago, RS; diuliagabert@hotmail.com

Letícia dos Santos Balboni; Universidade Regional Integrada do Alto Uruguai e das Missões- Campus de Santiago, RS; s.lbalboni@gmail.com

Natalia Pereira Araújo; Universidade Regional Integrada do Alto Uruguai e das Missões- Campus de Santiago, RS ; nataliaaraujo.uri@gmail.com

Patrícia Fonseca Martins; Universidade Regional Integrada do Alto Uruguai e das Missões- Campus de Santiago, RS; patimartins94@gmail.com

Claudete Moreschi; Universidade Regional Integrada do Alto Uruguai e das Missões- Campus de Santiago, RS; clau_moreschi@yahoo.com.br

\section{RESUMO}

Introdução: $\mathrm{O}$ enfrentamento da pandemia da Covid-19 necessita de atribuições essenciais dos profissionais de enfermagem, os quais, em tempos pandêmicos, precisam compreender a complexidade da saúde do idoso e focar na prestação de uma assistência qualificada. Objetivo: Identificar a produção científica existente acerca do cuidado de enfermagem a pessoa idosa em tempos de pandemia COVID-19. Métodos: Revisão integrativa da literatura, realizada na BDENF, em agosto de 2020, com os descritores "enfermagem" and "idoso" and "covid-19 or coronavirus". Foram incluídos artigos na íntegra, que abordam a temática pesquisada. Resultados: As três produções analisadas são artigos de reflexão, duas provenientes de periódicos nacional e uma dos EUA. Refletiram sobre o "Fortalecimento da enfermagem frente ao cuidado da pessoa idosa em tempos de COVID-19". Vislumbra-se que a pandemia poderá representar uma oportunidade de mudança nas relações com os idosos, mediante processos reformadores e transformadores, fortalecendo a enfermagem gerontológica. Os idosos são destaque na pandemia, sobretudo aqueles com doenças crônicas, despertando inúmeras reflexões, incertezas e questionamentos. Deste modo, urge a necessidade de restaurar a esperança e acreditar no cuidado gerontológico de enfermagem robusto, qualificado e seguro. A enfermagem precisa promover ações fundamentadas nas políticas e diretrizes atuais, bem como nos pilares da gerontologia, com manutenção da autonomia e independência do idoso. Conclusão: A enfermagem precisa ressignificar as ações de atenção ao idoso, buscando manter a autonomia e independência do idoso, melhorar a sua qualidade de vida, com foco no enfrentamento do momento pandêmico e vislumbrando cenários futuros de fortalecimento da enfermagem gerontológica.

Palavras-chave: Enfermagem; Idoso; COVID-19.

\section{Referências}

FILHO, J.J.M. et al. A saúde do trabalhador e o enfrentamento da COVID-19. Rev bras saúde ocup, v. 45, p. e14, 2020. SARAIVA, L.B. et al. Avaliação geriátrica ampla e sua utilização no cuidado de enfermagem a pessoas idosas. Journal of Health Sciences, v. 19, n. 4, p. 262-267, 2017. 